\title{
El redescubrimiento del trabajo de cuidados: algunas reflexiones desde la sociología
}

\author{
Pilar CARRASQUER OTO \\ Universitat Autònoma de Barcelona \\ Grup d'Estudis sobre Vida Quotidiana i Treball - QUIT \\ pilar.carrasquer@uab.es
}

Recibido: 05-07-12

Aceptado: $17-12-2012$

\section{RESUMEN}

Este artículo presenta algunas reflexiones sobre la (re)emergencia del trabajo de cuidados como objeto de estudio en la sociología contemporánea, en el contexto de la crisis de los cuidados, bajo el prisma de las desigualdades de género. Se argumenta que el debate en torno a los cuidados ha significado una puesta al día de las discusiones sobre el trabajo doméstico, la identidad y el lugar que las mujeres ocupan en la modernidad tardía. Pero no es sólo una puesta al día. Supone un cambio en el foco de interés, del trabajo a los cuidados, y la primacía del sujeto. Este cambio de enfoque presenta ventajas e inconvenientes para afrontar los retos que plantea la "crisis de los cuidados".

Palabras clave: cuidados, trabajo, género, desigualdad

\section{Rediscovering care work: some reflections from the Sociology}

\begin{abstract}
This paper presents some reflections on the (re) emergence of care work as object of study in contemporary sociology in the context of the crisis of care, through the prism of gender inequalities. It is argued that the debate on care has meant an update of the discussions on domestic work, identity and the place of women in late modernity. But it is not just an update. It represents a shift in focus of interest, from work to care, and the primacy of the subject. This shift in approach has advantages and disadvantages to confront the challenges arising from the "care crisis".
\end{abstract}

Keywords: care, work, gender, inequality

REFERENCIA NORMALIZADA

Carrasquer Oto, P. (2013). El redescubrimiento de cuidados: algunas reflexiones desde la sociología. Cuadernos de Relaciones Laborales, Vol. 31, núm.1, p. 91-113.

SUMARIO: Introducción. 1. El redescubrimiento del trabajo de cuidados. 2. La construcción de un campo de estudio. 3. En torno a la responsabilidad (social) de los cuidados. 4. Unos apuntes finales. 5. Bibliografía.

\section{El redescubrimiento del trabajo de cuidados}

Los cuidados se han hecho un hueco en la literatura académica. El concepto de cuidados forma parte de la producción científica en distintas disciplinas, aunque 
ello no significa consenso en cuanto a su definición y construcción como objeto de estudio (Letablier, 2007; Torns, 2008). El objetivo de este artículo es aportar algunas reflexiones en torno a la (re)emergencia de los cuidados como objeto de estudio en la sociología contemporánea ${ }^{1}$, en el contexto de la denominada crisis de los cuidados y bajo el prisma de las desigualdades de género.

Desde su aparición como preocupación académica en los años ochenta del siglo pasado hasta hoy, los cuidados en general y el trabajo de cuidados en particular, se han configurado como uno de los campos de estudio más vivaces y, de algún modo, controvertidos en la sociología contemporánea, como en otras disciplinas afines (Carrasco, Borderías y Torns, 2011). El trabajo de cuidados es la cara visible de la "querelle des sexes" (Prieto, 2007) en la modernidad, desde las aproximaciones que tienen en cuenta la dimensión de género en el análisis social, mientras que suele ser la cara oculta de los grandes relatos que han abordado las transformaciones en las sociedades occidentales de capitalismo avanzado, como los propuestos por Anthony Giddens o Zygmunt Bauman. En especial desde aquellos que acentúan el proceso de individuación como rasgo característico de esas sociedades y olvidan la trastienda sobre la que ese proceso se construye. Un trasfondo que la denominada "crisis de los cuidados" (Hochschild, 1995) ha contribuido a visibilizar. En buena medida, el redescubrimiento del trabajo de cuidados y su configuración como objeto de estudio se inscribe en el marco de ese nuevo escenario social de crisis de los cuidados, concepto que, en su versión inicial, alude a la tensión en la provisión de cuidados derivada del distinto papel de las mujeres en las sociedades desarrolladas de finales del Siglo XX (Hochschild, 1995). Y que en un sentido más amplio se refiere a los cambios en los contenidos, protagonistas y circunstancias en las que ese trabajo se da, como argumenta Amaia Pérez Orozco (2006). Crisis que, a su vez, se explica en el contexto más amplio de las transformaciones del capitalismo global en las sociedades de bienestar (Benería, 2011). Vista así, la problemática de los cuidados emerge como elemento nuclear para afrontar los retos teóricos y sociales que esas transformaciones plantean.

\subsection{Un cambio de escenario}

En efecto, en la acepción de Arlie R. Hochschild (1995), la crisis de los cuidados remite a la "stalled revolution" (Hochschild, 1989), la revolución estancada, protagonizada por las mujeres en las sociedades desarrolladas. Esto es, al hecho de que las mujeres han accedido al ámbito público, especialmente al empleo, sin menguar

${ }^{1}$ Este artículo tiene su origen en las Jornadas Los cuidados, entre el trabajo y la vida, celebradas en Madrid en diciembre de 2011, organizadas por EGECO, dentro del proyecto "Trabajo, cuidados, vida personal y orden social en el mundo de la vida de la sociedad española" (proyecto CSO2010-19450) y del proyecto "Producción Política de la categoría de cuidados: prácticas institucionales y experiencias de implementación en la atención a la dependencia" (proyecto FEM2010-18827). 
su protagonismo como cuidadoras en el ámbito doméstico. Lo que pondría en duda la viabilidad de la provisión de cuidados sustentada sobre el colectivo femenino, al mismo tiempo que supondría, como de hecho sucede, mayor trabajo para las mujeres. Un fenómeno que, en la década anterior, Laura Balbo (1978) había conceptualizado como "doble presencia", mostrando la ambivalencia de una realidad femenina que, lejos del ideal de superwoman, pone de manifiesto unas prácticas sociales y un "querer ser" que cuestionan el "deber ser" femenino ligado a domesticidad y al orden social que lo sostiene, en el sentido que Carlos Prieto (2000) da a esta expresión. La consolidación de la doble presencia femenina recompone la pauta de división sexual del trabajo propia de la primera modernidad. La doble presencia va a derivar en una mayor "carga total de trabajo" para las mujeres, según el concepto empleado por $\mathrm{M}^{\mathrm{a}}$ Ángeles Durán, y a plantear límites a la disponibilidad femenina hacia los cuidados, siendo uno de los indicadores que permiten evaluar las continuidades y cambios en la provisión de cuidados, entre familia-mercado-estado. Asimismo, la doble presencia configura una identidad femenina que no se construye sólo sobre los cuidados o el hogar-familia, sino también sobre el empleo. Su legitimación significa, al mismo tiempo, un lastre y una oportunidad para repensar las políticas de bienestar. Las propuestas teóricas y de intervención en clave de género van a compartir esa ambivalencia, para algunas autoras distintiva de la doble presencia (Borderías, 1996), amparadas en interpretaciones más o menos esencialistas de la relación entre género y cuidados, hasta hoy.

Esta identificación inicial de la crisis de los cuidados ligada al fenómeno de la doble presencia femenina, sigue siendo un diagnóstico certero de la problemática. Pero su contenido se ha completado con la consideración de otros factores que contextualizan y enriquecen su alcance y contenido. Así, la crisis de los cuidados se ha convertido en un concepto heurístico que llama la atención sobre los cambios demográficos, sociales, pero también económicos y políticos, observados en las sociedades desarrolladas en estas últimas dos décadas y su incidencia en las necesidades y en la provisión de cuidados (Rodríguez Cabrero, 2011a). Entre ellos destaca, en primer lugar, la "revolución reproductiva" (McInnes y Pérez Díaz, 2008). El alargamiento de la esperanza de vida y el envejecimiento de la población, junto a una mayor "eficiencia reproductiva", permiten el mantenimiento del volumen de población, a pesar de la disminución en la fecundidad. Sin embargo, por más que vivir más tiempo no signifique necesariamente vivir de manera menos autónoma, como muestra el nuevo papel de las personas mayores como cuidadoras (Agulló y Martín Palomo, 2011), no es menos cierto que la inversión de la pirámide demográfica en las sociedades desarrolladas, supone un incremento de las necesidades de cuidados, que no pueden circunscribirse a la crianza de los hijos e hijas, a pesar de que esa consideración pueda seguir presente en el imaginario colectivo (Lewis, 2009). El cuidado de las personas mayores dependientes se perfila como una necesidad perentoria en Europa (Cameron y Moss, 2007) y en España (Sarasa, 2003, 2004; Durán, 2005, 2011; Rodríguez Cabrero, 2011b; Abellán, Esparza y Pérez Díaz, 2011). A ello hay que añadir los cambios en la consideración del significado de cuidar y en las expectativas en relación a la calidad de los cuidados, tanto para 
los niños y niñas (Brannen, Moss y Mooney, 2004) como para las personas adultas dependientes o discapacitadas (Puga y Abellán, 2004). En este sentido, en segundo lugar, la crisis de los cuidados tiene que ver con los cambios en las formas familiares y en los roles e identidades de género. Un fenómeno ampliamente abordado en España por especialistas como Inés Alberdi, Constanza Tobío o Lluís Flaquer. Y que se ha concretado en el estudio de los cambios en las formas y protagonistas de los cuidados (Tobío, 2012). En tercer lugar, la crisis de los cuidados evoca un proceso de cambio social más amplio, ligado al cuestionamiento del estado de bienestar, allí donde éste se ha dado, y de erosión de la norma social de empleo (Prieto, 2000, 2002), que la actual crisis no hace más que exacerbar. En relación al primer aspecto, valga como ejemplo la deriva de uno de los principales instrumentos previstos en España para afrontar las necesidades de cuidados, la conocida como Ley de dependencia de 2006, a cuyas insuficiencias de origen se añade su limitado desarrollo ${ }^{2}$. En cuanto al segundo, las transformaciones socio-productivas que algunos han sistematizado bajo conceptos como "sociedad del riesgo" (Beck, 1992), no sólo tienen como resultado la degradación del empleo y de las garantías a él asociadas. Introducen incertidumbre y heterogeneidad en las trayectorias laborales y vitales de ambos géneros, de modo que la combinación entre empleo y trabajo de cuidados a lo largo del curso de vida deviene un nuevo foco de tensión, sobre todo para las mujeres en situación de doble presencia. En definitiva, la crisis de los cuidados remite a todo un conjunto de fenómenos que ponen en entredicho el statu $q u o$ en torno a las necesidades y a las formas de provisión de cuidados, propio de unas sociedades sustentadas en el modelo ganapán/ama de casa.

\subsection{Nuevos y viejos retos en el abordaje del trabajo de cuidados}

En este contexto, el debate actual en torno a los cuidados supone, en cierto modo, una puesta al día de las discusiones, propuestas y elaboraciones sobre el trabajo, la identidad y el lugar que ocupan las mujeres en la modernidad tardía. Bajo este prisma, el trabajo de cuidados sería hoy lo que a mediados de los años setenta del

${ }^{2}$ Las expectativas suscitadas por la Ley de dependencia fueron puestas a prueba de inmediato. Como ejemplo, ya en 2006, la revista Lan Harremanak.Revista de Relaciones Laborales dedica un monográfico titulado "El debate sobre la dependencia", a discutir los límites y los sesgos que dicha Ley planteaba, tanto desde el punto de vista de la definición de dependencia o de autonomía personal, como de las actuaciones contempladas y de su regulación. Entre las actuaciones, la figura de "cuidadores profesionalizados" es una de las apuestas controvertidas de la Ley, dada la naturaleza especial de un trabajo que no es ni empleo ni trabajo doméstico y familiar, así como, por su potencial efecto de refuerzo de la feminización del cuidado en el hogar, como advertía Carmen Bravo en el XVIII Taller del Fórum de Política Feminista, celebrado en 2008. El escaso desarrollo de servicios públicos de atención a la vida diaria, junto a los recortes y restricciones en el acceso al cuidado de la dependencia, corroboran algunas de las reservas iniciales. 
siglo pasado fue el trabajo doméstico: una oportunidad para afrontar cuestiones de gran calado tanto académico como social. En el debate sobre los cuidados es posible identificar problemáticas y argumentos que arrancan con los intentos de conceptualización del trabajo doméstico, como la visibilidad del conjunto del trabajo femenino, la contribución del trabajo doméstico y familiar al bienestar y a la reproducción y sostenibilidad de la vida humana, la construcción sexuada del orden social o la división social y sexual del trabajo. Y, tan o más relevante, comparte con aquél el protagonismo que el feminismo y las especialistas feministas, plurales en sus aproximaciones y propuestas, tiene en su origen y desarrollo como objeto de estudio (Carrasco, Borderías y Torns, 2011). De ahí la idea de redescubrimiento en el título que encabeza estas páginas. Sin embargo, toda esa temática nuclear en la discusión sobre los cuidados se ha visto revisada y ampliada hacia otros territorios, hasta el punto que, en la actualidad, los cuidados constituyen un campo de investigación con entidad propia, siendo abordados no necesariamente aunque sí de forma más acusada, desde el feminismo. Las principales líneas de continuidad tienen que ver con el hecho que el análisis de los cuidados, en particular aquellos desarrollados bajo la óptica del trabajo de cuidados, encuentra un sólido punto de partida en los esfuerzos de elaboración y de medida del trabajo doméstico y familiar (Torns, 2008) y en la bondad de ese concepto para explicar las desigualdades, la identidad y las relaciones de género. Las divergencias se observan en el acento que la vertiente relacional, afectiva y emocional adquiere en la definición de los cuidados y del trabajo de cuidados. Lo que, por otra parte, abre la posibilidad de delimitar e interpretar esa parcela del trabajo doméstico como una actividad de naturaleza distinta (Himmelweist, en Carrasco, Borderías y Torns, 2011), y vincularla a la problemática de actividades y servicios de cuidados profesionalizados y remunerados. Esto es, permite ubicar el foco de atención inicial en la definición de los cuidados, entendidos como aquellos proporcionados por las mujeres en el hogar-familia, en el marco más amplio de las necesidades de atención a la vida humana propias de las sociedades de bienestar. Como contrapartida, el acento en esa dimensión afectivoemocional tiende a reorientar el interés hacia el sujeto protagonista de la provisión de cuidados, las mujeres, en detrimento, a veces, de una lectura de los cuidados como trabajo. Dando, así, un nuevo relieve a la tensión, ya latente en relación al trabajo doméstico, entre la lógica de la redistribución y la lógica del reconocimiento que preside el análisis del trabajo femenino (Fraser, 1994, 2000).

En definitiva, el trabajo de cuidados sigue siendo "a woman-specific concept" (Daly y Lewis, 2000:283), pero el interés por ese trabajo se ha ampliado hacia otros puntos de vista, otras problemáticas y otros colectivos. La revisión del concepto de dependencia y su relación con el de cuidados (Fine y Glendinning, 2005), los interrogantes sobre la provisión de cuidados y su profesionalización fuera del marco institucional y normativo del hogar-familia, la importancia de la relación entre prestación y recepción de cuidados, o sobre los costes materiales, pero también afectivos o emocionales del cuidado, así como su distribución entre los distintos grupos e instituciones sociales, han ampliado el horizonte del debate. En esta ampliación, la consideración de los cuidados como eje definidor de los modelos de 
bienestar ha jugado un papel decisivo (Daly y Lewis, 2000). La propia idea de ciudadanía y los derechos y deberes que la acompañan también se ha visto revisada (para una síntesis, véase los trabajos presentados en el Congreso Internacional organizado por Emakunde, SARE.2004. ¿Hacia qué modelo de ciudadanía? (VVAA, 2005), en particular los de Bárbara Hobson (2005), Chiara Saraceno (2005) y Joan Tronto (2005), allí presentados).

\section{La construcción de un campo de estudio}

Los cuidados aluden a lo femenino, a la intimidad y a la privacidad. Se perciben como una obligación moral que parece que sólo atañe a las mujeres. Suelen tener una connotación positiva de entrega y altruismo, que puede ser vivida como fuente de placer y de satisfacción personal (Lagarde, 2004). La orientación hacia los cuidados es aparentemente innata, así como también parecen innatos los conocimientos y habilidades necesarios para su desarrollo. Los cuidados remiten a las pequeñas y grandes atenciones que las mujeres llevan a cabo para el bienestar de los miembros del hogar. Pero responden a un imperativo social cuyo objetivo, la reproducción y la sostenibilidad de la vida humana (Carrasco, 2001; Pérez Orozco, 2006), favorece la naturalización y la ocultación de esa actividad y de quien la lleva a cabo. Todo un conjunto de imágenes que configuran los cuidados como un territorio eminentemente femenino, como una actividad que se mueve entre el placer y la obligación moral, entre la necesidad social y la responsabilidad individual y colectiva. Conformando algunos de los lugares comunes en torno a los cuidados que más de veinte años de reflexión e investigación sobre el tema han contribuido a reforzar o a cuestionar.

Desde las corrientes feministas anglosajonas pioneras en el abordaje de esta temática, la construcción teórica de los cuidados va a estar caracterizada por dos grandes cuestiones, de distinto rango. Por un lado, el de su viabilidad dentro de la corriente principal de sus respectivas disciplinas. Por otro, el de la dualidad como rasgo típico de los cuidados. En el primer caso, construir la posibilidad de abordar el estudio de los cuidados va a significar un esfuerzo de revisión de los conceptos y categorías de análisis que, en el límite, supone una ruptura con el corpus teórico comúnmente aceptado, en una lógica similar a la observada en relación al concepto de trabajo (Carrasco, Borderías y Alemany, 1994). Desde algunos puntos de vista, esta ruptura sería la única forma viable de encarar el proceso de deconstrucción y reelaboración de unas categorías de análisis androcéntricas, que sólo pueden ser revisadas desde el exterior y en paralelo a la producción científica ciega al género (Braidotti, 2000, 2004). Se trata de hacer visible lo invisible y de legitimarlo como objeto de estudio, con una mirada propia. Por otro lado, una dualidad que atraviesa la idea de cuidados, en cualquiera de sus dimensiones. La principal es la dualidad en cuanto a su naturaleza (trabajo vs. amor y/o emociones), pero también con respecto a las relaciones sociales que lo definen (formal vs. informal; remunerado o no; de reciprocidad, en sus distintas manifestaciones, vs. poder o dominación) o en 
relación al espacio social en que se desarrollan (público vs. privado). Los desarrollos recientes continúan impregnados de esa impronta inicial (England, 2005).

\subsection{La dualidad de los cuidados: un atributo persistente}

Hilary Graham (1983) plantea esta naturaleza dual de los cuidados en su obra Caring: a Labour of Love, reconocida como uno de los textos pioneros en la construcción teórica de los cuidados. Los cuidados se definen a través de las dos dimensiones reflejadas en su título. Los cuidados son trabajo, el que se lleva a cabo por las mujeres en el hogar- familia para la reproducción de sus miembros. Y emociones, afecto, amor. Un tipo de relación propio de una actividad basada en las relaciones interpersonales, que se desarrolla bajo el signo de obligación moral para las mujeres y que tiene como núcleo la maternidad. Esta definición restrictiva de los cuidados va a ser contestada, desde el inicio, por los estudios procedentes de la política social, que muestran como los cuidados no se circunscriben al hogar, ni se orientan sólo a la crianza o al cuidado de las personas adultas sanas del entorno familiar. Roy Parker (1981) y Clare Ungerson $(1983,1990,2005)$ abren el concepto de cuidados hacia la dependencia y hacia los cuidados, informales o no, prodigados en la esfera pública. Para esta última, la dimensión afectivo-relacional de los cuidados no es exclusiva los cuidados informales en el hogar y forma parte de los cuidados formales provistos en los servicios socio-sanitarios, de modo que, desde este punto de vista, la distinción entre cuidados formales e informales carece de sentido. Tal como señala Carol Thomas (1993), la propia Graham (1991) revisará el concepto de cuidados para incluir los "servicios substitutivos" que de manera remunerada prestan los no familiares, también por lo común mujeres, en el hogar. Una ampliación que permite mostrar cómo los cuidados tienden a ser patrimonio del género femenino, pero atravesado por el origen étnico y social. Una tríada que hoy reemerge en relación a los procesos migratorios vinculados a la provisión de cuidados (Parella, 2003), en el capitalismo global.

En paralelo, la "ética del cuidado" (Gilligan, 1982) surge como contrapuesta o complementaria a la "ética de justicia", en un intento de hacer ver las distintas lógicas que atraviesan los cuidados y la producción de bienes y servicios en el mercado, y de hacer valer la existencia de otro tipo de vínculos y de relaciones que encuentran en la singularidad de los cuidados la fuente de su experiencia (Benhabib, 1992). Las nociones de reciprocidad, de don, como identificadoras de la relación de cuidados, van a sumarse a las cualidades que a priori parecen ser propias del género femenino como la entrega, la abnegación o el altruismo (Legarreta, 2008; Martín Palomo, 2008). Los cuidados conforman el núcleo de la intimidad femenina. Las mujeres, "revolucionarias emocionales de la modernidad" (Giddens, 1992:130), abren el camino al desarrollo del espacio social de la intimidad, gracias a un aprendizaje como cuidadoras del hogar-familia, avalado por la ideología de la domesticidad burguesa. La intimidad pasa a ser el espacio expresivo de las mujeres, a través de su dominio de las relaciones emocionales y afectivas, dentro de la separación de esferas y sujetos que la división sexual del trabajo define. Las mujeres no descubrirán el "ámbito íntimo" (Béjar, 1988) como refugio frente a los retos de la moderni- 
dad, pues ese sería ya su espacio. En este sentido, sin embargo, la problematización de la "vida privada" aportará otros matices. El hogar, espacio privado por excelencia, podrá ser visto como el foco de la actividad femenina, aunque dicha actividad, el trabajo doméstico y de cuidados, no se reconozca como trabajo ni genere derechos de ciudadanía. En clave de género, el espacio privado no puede construirse como espejo del espacio público. Para las mujeres, el ámbito privado es el ámbito de la "privación de sí" (Murillo, 1996). Una noción que define a la perfección el trabajo de cuidados, en particular cuando este se refiere a los cuidados que las mujeres desarrollan de manera no remunerada en el hogar-familia.

A pesar de estos esfuerzos, estos planteamientos iniciales no permiten una comprensión global del trabajo de cuidados, al tiempo que parecen reforzar la idea de una especie de "eterno femenino", basada en la confusión de presuponer que la abrumadora responsabilidad femenina en el trabajo de cuidados responde al carácter sexuado de éstos, en sentido esencialista (Fraser, 1994; Crompton, 2006). Una apreciación sobre la que Martha Nussbaum ya había llamado la atención al señalar que el amor y los cuidados a menudo se tratan como si existiesen de manera natural, en lugar de reconocer el papel de la costumbre, la ley y las instituciones en la formación de las emociones (Nussbaum en Crompton, 2006:17). Un envite que, en el caso español, han recogido estudiosas como Soledad Murillo (2004) o $\mathrm{M}^{\mathrm{a}}$ Jesús Izquierdo (2004), entre otras.

\subsection{La idoneidad de un concepto global de trabajo de cuidados}

En el entorno anglosajón, la reflexión en los años noventa permite dar un giro a estas limitaciones. Destacan dos propuestas distintas en las que se reconocen buena parte de las actuales aproximaciones al trabajo de cuidados: el concepto de cuidados propuesto por Carol Thomas (1993) y el de "social care", de Mary Daly y Jane Lewis (2000), ambos disponibles en castellano (Carrasco, Borderías y Torns, 2011). La revisión de las propuestas sobre el concepto de cuidados, permite a Thomas identificar tres grandes líneas de estudio sobre la cuestión: la del trabajo de cuidados para el conjunto de miembros del hogar, realizado por las mujeres de manera no remunerada, es decir, el trabajo doméstico-familiar; la de la naturaleza de esa actividad y su relación con la identidad femenina y la del estudio de la atención de las personas dependientes (dentro o fuera del hogar) como preocupación ligada a la política social. Para la autora, esta pluralidad de intereses y objetivos en torno a los cuidados, impide una aproximación completa a los mismos. Lo que plantea un doble reto: la elaboración de un concepto integrado de cuidados y garantizar la solidez teórica del mismo. En cuanto al primero, Thomas propone un concepto de cuidados delimitado mediante siete dimensiones: la identidad social de la persona cuidadora, la identidad social de la persona receptora de los cuidados, las relaciones interpersonales entre la persona cuidadora y la receptora de los cuidados, la naturaleza de los cuidados, el dominio social (público o doméstico) en que se producen, la relación económica que media en la provisión de cuidados y el contexto institucional en que se desarrollan. A pesar de las críticas que ha podido suscitar (Letablier, 2007), la propuesta es esclarecedora. Supone una dimensionalización cabal y articu- 
lada del trabajo de cuidados que permite superar las lecturas parciales del mismo y proporciona un entramado analítico que hace posible una aproximación global y comprensiva a los cuidados, como ilustra la investigación de Cristina Vega (2009), en el caso español. Sin embargo, la propia autora reconoce que se trata de un concepto "empírico" que no tiene suficiente capacidad explicativa y que no alcanza a delimitar un campo de estudio, en el sentido que sugiere la propia M. Thérèse Letablier (2007). Ese será uno de los logros del concepto de "social care" propuesto por Daly y Lewis (2000), unos años después.

En efecto, el concepto de "social care" representa uno de los intentos más exitosos de salir de ese impasse y dotar de contenido teórico al análisis de los cuidados, o del trabajo de cuidados, pues es así como ambas especialistas definen esa actividad. A diferencia de Thomas, el objetivo de Daly y Lewis es proponer un concepto de cuidados que, sin obviar su capacidad explicativa de la vida de las mujeres, permita afrontar el análisis de los estados de bienestar y sus cambios. Esto es, salir de una visión de los cuidados focalizada en "lo femenino" y trasladarla hacia la discusión sobre las necesidades y la provisión de bienestar. En esta propuesta, los cuidados son un trabajo socialmente organizado en el que participan distintos grupos, instituciones y espacios sociales, que se articula y distribuye de acuerdo a distintos patrones socio-culturales y económicos. El trabajo de cuidados deviene una pieza clave en la relación estado-mercado-familia, desde una perspectiva dinámica capaz de aprehender los cambios y continuidades en los distintos arreglos institucionales en torno a los cuidados. Familia -estado-mercado conforman el triángulo socioinstitucional en que se desarrollan los cuidados, cuyo denominador común son las mujeres.

No es de extrañar, en este sentido, que esta lectura haya encajado a la perfección con los debates sobre los regímenes de género y de bienestar (Pocock, 2005), las políticas sociales o de protección social (Letablier, 2007), en un contexto de cambio sociodemográfico, y donde esos regímenes de bienestar y de protección social basados en el modelo "ganapán/ama de casa", parecen derivar hacia un modelo de "two adults workers", más ideal que real (Annesley, 2007, Lewis, 2009; Daly, 2011). En una aproximación macrosocial y de investigación comparada, la organización social del cuidado se sintetiza en la idea de "regímenes de cuidado" (o "modelos de cuidados"), bien sea para explorar su relación con los "modelos de empleo" (Simonazzi, 2007), bien para ubicar la problemática de las "cadenas de cuidado", especialmente en los países "familistas" del sur de Europa (Bettio, Simonazzi y Villa, 2006), o para abordar los retos de la profesionalización del trabajo de cuidados (Recio, 2010). Una visión que tiene su correlato en lecturas que enfatizan la dimensión cultural de los regímenes de género (Pfau-Effinger, 1998) como elemento indispensable para comprender los arreglos institucionales en relación a los cuidados, a pesar de que, a menudo, ese tipo de perspectivas puedan obviar la dimensión material de los mismos (Crompton, 2006). 


\subsection{El trabajo de cuidados en la vida cotidiana}

El cuadro de este proceso de construcción de un campo de estudio sobre los cuidados quedaría incompleto sin una mención al trabajo de cuidados en la vida cotidiana. Un escenario que permite explorar el carácter transversal del trabajo de cuidados, en la línea planteada por Thomas (1993), capaz de trascender el binomio público/privado. La vida cotidiana se propone como un escenario adecuado para explorar cómo se gestiona y se lleva a cabo ese trabajo, teniendo en la experiencia femenina de la doble presencia el marco adecuado para ello. Un territorio sobre el que especialistas italianas y francesas han centrado su atención, de manera menos visible y en paralelo al debate anglosajón de los años ochenta y noventa. Entre ellas destacan dos obras pioneras: Éspace et temps tu travail domestique, publicado en 1985 por Danielle Chabaud-Rychter, Dominique Fougeyrollas-Schwebel y Françoise Sonthonnax, del grupo GEDISST, y Time to care, editado por Balbo, en 1987.

El trabajo de cuidados es un rasgo de la vida cotidiana de las mujeres. Mediante ese trabajo, las mujeres proporcionan bienestar cotidiano (Moreno, 2007). Los estudios sobre la doble presencia (Balbo, May y Micheli, 1990) han abordado esta cuestión, propiciando una visión de la vida cotidiana como espacio social en el que se articula el trabajo de cuidados entre los distintos protagonistas e instituciones, y se dirimen los conflictos derivados de tal actividad (Bimbi y Capecchi, 1986). Desde esta óptica, la vida cotidiana podrá interpretarse como un espacio que actúa como amortiguador de las tensiones que genera la propia reproducción social, desde el punto de vista del trabajo de cuidados.

El tiempo emerge como dimensión básica (Balbo, 1987). No en vano, para Helga Nowotny (1992) la vida cotidiana ya no es el espacio de la rutina salpicado por acontecimientos más o menos predecibles. Es el espacio de la confrontación entre los distintos órdenes temporales, fijados de manera dominante por el mercado y el estado, y la posibilidad de un tiempo "privado", de un tiempo "para sí" que, sin embargo, escapa a la cotidianidad femenina de la doble presencia (Nowotny, 1987). Más bien, el problema cotidiano que el trabajo de cuidados va a plantear a las mujeres en situación de doble presencia es el del "encaje temporal" (Lewis y Weigert, 1992) de tiempos y actividades que las mujeres viven en clave sincrónica y no diacrónica. Una dificultad agravada por la naturaleza del tiempo de trabajo de los cuidados y por la progresiva desincronización de esos tiempos y actividades en el post-fordismo. El tiempo del trabajo de cuidados es difícil de percibir y de delimitar (Ramos, 2011). Su ritmo y su intensidad dependen tanto de factores que se podrían calificar de internos (número de personas que conforman el hogar y sus características) o externos (ritmos productivos, disponibilidad y accesibilidad al soporte público o privado de los cuidados), con distintas exigencias a lo largo del curso de vida. Es un tiempo plagado de rutina pero también de imprevistos, de flexibilidad y, al mismo tiempo, de rigidez (Carrasquer; Torns, Tejero y Romero, 1998), de tal manera que el "encaje temporal" constituye una ardua tarea de gestión cotidiana para las mujeres, agravada por los cambios en la organización socio-temporal del trabajo productivo (Del Re, 1995; Torns, 2001, Callejo, Prieto y Ramos, 2008). El tiempo de los cuidados y el tiempo para los cuidados, se plantean en clave de ciudadanía. 
De ahí que se reclamen unas políticas de tiempo que conjuguen esas distintas realidades socio-temporales, sin profundizar en las desigualdades entre e intra género (para una síntesis del debate, véase Torns, 2004, 2007; Borràs, Torns y Moreno, 2007).

La vida cotidiana es también el escenario donde se entretejen las estrategias en torno a los cuidados y a la gestión de los mismos. Este es uno de los aspectos más interesantes de la propuesta de las sociólogas del GEDISST mencionadas. En su estudio indagan sobre las estrategias que las mujeres llevan a cabo para armonizar las necesidades cotidianas de cuidado, según las restricciones espacio-temporales que imponen los distintos contextos institucionales implicados, y la propia disponibilidad, limitada por actividad laboral. La línea femenina hijas-madres-abuelas hilvana la provisión y gestión cotidiana del trabajo de cuidados. Pero mostrarán como esas estrategias, al igual que la propia percepción del trabajo de cuidados, difieren según la clase social. Además, en su interpretación, plantearán que, más que la idea de soporte altruista, lo que surge en esas relaciones son relaciones de poder, de obligación de cuidar en el futuro, a cambio de soporte en las necesidades de cuidado en el presente. Un tipo de relación que podría ser vista como reproductora de la división sexual del trabajo. Pues el conflicto que emerge del trabajo doméstico, en general y del trabajo de cuidados, en particular, permanecería soterrado en el ámbito privado y se plantearía dentro del colectivo femenino y no como un conflicto entre géneros, o como demanda de ciudadanía. Una interpretación que contrasta con las dominantes, en aquel momento, en el debate anglosajón, tal como se ha señalado, y que permite explorar una de las dimensiones significativas en torno al "malestar" de los cuidados.

\section{En torno a la responsabilidad (social) del cuidado}

Las mujeres son las principales cuidadoras en las sociedades de bienestar, donde la división sexual del trabajo persiste sin apenas cambios (Crompton, 2006; Lewis, 2009; Gálvez, Rodríguez y Domíngez, 2011). Así mismo, frente a las teorías de la individualización que parten de considerar la existencia de un corte nítido entre las formas o estilos de vida del presente y los del pasado, otras voces argumentan que es posible observar líneas de cambio y de continuidad (Duncan, 2011). Este autor constata no sólo que las personas no siempre priorizan su propio proyecto de vida en términos de satisfacción personal, sino que la "destradicionalización" de algunas prácticas sociales convive con la existencia de otras bien arraigadas. Como ejemplo menciona la evidencia de cómo la pérdida de importancia del matrimonio frente a la cohabitación no significa una mutación en determinadas prácticas cotidianas en relación al papel de "madres y esposas" adjudicado a las mujeres. El "nuevo orden de género" (presencia femenina en el mercado laboral, cambios en las formas de familia, mayor control sobre la propia sexualidad, igualdad formal entre mujeres y hombres) matiza pero no invalida esas constataciones, ni en relación al trabajo de cuidados en y para el hogar-familia, ni en relación a quien se ocupa principalmente 
de ese trabajo de manera remunerada y las condiciones en que ese trabajo se desarrolla (Tronto, 2004; Benería, 2011).

La identificación del trabajo de cuidados con el género femenino marca el curso de la discusión en torno a la responsabilidad social de los cuidados y de las propuestas para superar la invisibilidad de esa contribución femenina al bienestar y a la reproducción social, así como su desigual distribución entre géneros, o entre las propias mujeres (Fraser, 1995). La ambivalencia entre trabajo y emociones reemerge para explorar posibles vías alternativas (VVAA, 2005). ¿Cómo resolver el imperativo social del trabajo de cuidados sin profundizar en las desigualdades y jerarquías sociales que lo acompañan? ¿Qué resistencias para propiciar otras formas de organización social del mismo? Nicole Mosconi y Marion Paoletti (2011) plantean estas cuestiones en el apartado "Controverse", de la revista Travail, genre, societés, bajo un contundente lema: "Le care: projet égalitaire ou cache misère?".

Desde la filosofía, esta cuestión se ha planteado en torno a la discusión sobre si los cuidados competen al mundo de la "vida buena" (el espacio de la autonomía o de la elección personal) o interesan al ámbito de la moral y, en concreto, al ámbito "público" de la justicia y, de ser así, ¿a qué justicia? En ese campo, una de las vías para afrontar esos interrogantes es la sugerida por Seyla Benhabib (1992). A su juicio, la "ética de los cuidados" y la "ética de la justicia" no son contrapuestas sino complementarias. Para Benhabib, en relación a los cuidados, la cuestión que se plantea es, más bien, "cómo circunscribir el ámbito de la autonomía individual (tanto legal como políticamente) en el que se ejercitan las elecciones que competen a formas diversas de la vida buena de manera tal que sea compatible con los principios universalistas de justicia" (Benhabib, 1992:61, nota 21). No se trata de una cuestión menor. La posibilidad de intervenir de manera aceptable en un ámbito considerado "privado" y, como tal, circunscrito a la discrecionalidad de cada cual, planea sobre las posibles formas de organizar socialmente el cuidado (Tronto, 2004). Entonces, desde el punto de vista de los cuidados, ¿qué se entiende por la vida buena? ¿Qué se considera adecuado? ¿Cómo se perciben hoy los cuidados? Pues tal como recuerdan Daly y Lewis (2000), los cuidados se inscriben en un marco normativo de responsabilidad y obligación con respecto a quién debe cuidar, cómo y quién debe ser cuidado. Este marco normativo conformaría las distintas formas de la vida buena aceptables en una determinada sociedad. Esas normas impregnan la organización social del cuidado y contribuyen a explicar sus características y las resistencias al cambio. Explorar las prácticas, las representaciones y las resistencias en torno al trabajo de cuidados permite intentar responder a unos interrogantes que, en términos sociológicos y políticos, se proponen en torno al bienestar y a la ciudadanía.

\subsection{Prácticas, representaciones, resistencias}

Además de mostrar la desigual distribución del trabajo de cuidados, sobre todo en razón de género, investigaciones recientes como las que se detallan a continuación, han abordado la cuestión de las prácticas, representaciones y resistencias en torno al trabajo de cuidados, desde perspectivas que buscan explorar la heteroge- 
neidad y el contraste en las formas de hacer y de percibir, los cambios y las resistencias con respecto al trabajo de cuidados. Contribuyen, de ese modo, a poner en evidencia cómo esas prácticas y esas percepciones tienen que ver con los diferentes significados que puede tener la idea de cuidados y con el valor, reconocimiento y obligatoriedad con los que se identifique ese trabajo.

Explorar la relación entre mujeres y cuidados desde el punto de vista de las normas y valores sociales que explican, legitiman y reproducen el protagonismo femenino en los cuidados, tiene en la naturaleza de los cuidados y en la identidad femenina un terreno propicio para su interpretación. Los procesos de socialización, el papel de las mujeres en la reproducción o la propia naturalización de los cuidados, son algunos de los argumentos considerados (Izquierdo, 2004; Martín Palomo, 2008 o Tobío, Agulló, Gómez y Martín Palomo, 2010). Sin embargo, la investigación en este campo ha puesto de manifiesto la heterogeneidad de las prácticas y de las cosmovisiones en torno a los cuidados y a la obligación de cuidar entre las propias mujeres, que escapan a la lógica de una explicación esencialista. Así, las mujeres asumirían el trabajo de cuidados pero no todas del mismo modo ni con la misma intensidad o el mismo sentido de la obligatoriedad. Estudios recientes abundan en esa dirección para el caso español (Moreno, 2007; Vega, 2009; Martín Palomo, 2010; Tobío, Agulló, Gómez y Martín Palomo, 2010), en línea con los de otras latitudes (Brannen, Moss y Mooney, 2004; McDowell, Ray, Perrons, Fagan y Ward, 2005; Compton, 2006; Crompton y Lyonette, 2010).

Esos estudios también ponen de manifiesto la idoneidad de abrir el análisis de los cuidados hacia otros ejes de desigualdad y de estratificación social, así como hacia el gran ausente del trabajo de cuidados, el género masculino. La tensión tiempo de trabajo productivo y tiempo de cuidado es un modo de hacer visible la distinta percepción del trabajo de cuidados entre hombres y mujeres (Prieto, 2004; Callejo, Prieto y Ramos, 2008). La presencia masculina en los cuidados empieza a superar lo anecdótico (Tobío, 2012). Pero los hombres tienden a percibir el trabajo de cuidados en su vertiente afectiva y no como un trabajo obligado y, en general, como una actividad que no entorpece (ni debe) la actividad laboral (Dermott, 2005; Borràs, Moreno y Recio, 2009). Por su parte, también se observan resistencias femeninas a ceder poder doméstico, en particular en relación a la maternidad y a la crianza; la maternidad y la paternidad compartidas aparecen como posibles sólo en determinados perfiles sociales, las clases medias urbanas, donde ninguna de las dos figuras se asocia a una presencia continuada con los hijos o las hijas (Torns, Borràs, Carrasquer, 2003/04). El sentido de la obligatoriedad o del deber de cuidar, se expresan de distinta forma. Cuidar puede significar velar por, acompañar, estar disponible para, o, por el contrario, realizar todas las tareas que el cuidado de una persona implica. Según la clase social, el sentido de la obligación (o del propio espacio social) se puede traducir en considerar una injerencia el soporte externo hacia esa tarea entre las mujeres (Vega, 2009; Castelló, 2011). A pesar del camino recorrido, queda mucho por explorar en relación a cómo perciben los cuidados y el trabajo de cuidados las personas que los reciben. 


\subsection{Los cuidados como derecho y deber de ciudanía}

Los cuidados se han situado en el centro de los debates sobre el estado de bienestar. El trabajo de cuidados se inscribe en y se provee desde el entramado estado- mercado -familia. La propia construcción de los estados de bienestar no es ajena a cómo se entienden las necesidades de cuidados y a cómo deben satisfacerse. La ampliación de esas necesidades hacia los cuidados de la dependencia y la discapacidad pone a prueba la idea de un trabajo de cuidados sustentado sobre el trabajo femenino no remunerado en el hogar- familia, de acuerdo a los cánones de la ideología de la domesticidad y de la división sexual del trabajo. La doble presencia femenina tensiona un orden socio-temporal y de organización del trabajo total, que ya no responde a la figura de una proveedora de cuidados siempre disponible. Sin embargo, las mujeres son las protagonistas del trabajo de cuidados en cualquiera de las modalidades en que este trabajo se da. La consideración de los cuidados como derecho de ciudadanía vehicula la idea de que la responsabilidad social de los cuidados es colectiva y no individual, pero no garantiza que esa responsabilidad se distribuya de manera más equitativa entre los distintos grupos sociales (Tronto, 2005). Las palabras de Saraceno sobre este particular, resultan esclarecedoras:

Una ciudadanía con género no significa desarrollar modelos de ciudadanía diferentes para hombres y para mujeres, ni reconocer distintas bases para los derechos que de ella se deriven. Más bien significa ampliar esas bases para acomodar un mayor conjunto de necesidades (y capacidades) a la vez que se descongela la rígida división por géneros del trabajo y de las responsabilidades (Saraceno, 2005:2).

Para Saraceno, ello implica revisar el "contrato de género" (Paterman, 1995), siendo el trabajo de cuidados uno de los elementos nucleares del mismo.

Ahora bien, desde el punto de vista de los cuidados y del trabajo de cuidados, su viabilidad como derecho y deber de ciudadanía también pasa por revisar qué significan los cuidados y por reconsiderar ambas caras de lo que algunas consideran la misma moneda: ese derecho y ese deber de cuidar (Glenn, 2000; Tronto, 2005). Sólo de este modo será posible superar la ecuación trabajo de cuidados - mujeres desigualdad. Como paso previo, ello significa reconocer el carácter de trabajo de los cuidados, reconocer como miembros de pleno derecho, con voz como ciudadanas y ciudadanos y con acceso a los recursos necesarios, a las personas que reciben los cuidados, así como reconocer socialmente el trabajo de cuidados que las personas llevan a cabo. Lo que tiene como corolario, legitimar los cuidados como responsabilidad pública, facilitar el acceso igualitario a los cuidados, evitar que el peso de los cuidados recaiga sólo sobre los colectivos menos favorecidos (Glenn, 2000:88). Salir del "círculo vicioso" de los cuidados (Tronto, 2005) exige plantear la relación entre prestación y recepción de cuidados como algo en lo que todo el mundo está implicado. Y reconsiderar los límites entre lo que cada cual entiende como ideal de vida buena (cómo imagina que quiere ser cuidado o cuidada) y una idea de prestación de cuidados basada en el carácter altruista de esa prestación. En definitiva, "requiere que el cuidado sea considerado una condición humana, parte de nuestros 
valores públicos y parte de nuestra forma de comprender qué es y hace la ciudadanía" (Tronto, 2005: 250).

\section{Unos apuntes finales}

La crisis de los cuidados ha propiciado la discusión en torno a un trabajo imprescindible y desigualmente repartido. Los cuidados y el trabajo de cuidados siguen suscitando controversia en cuanto a su definición, en un debate que excede el terreno académico. Pero ello no ha impedido avanzar en la delimitación de un campo de estudio sociológico de crucial interés en las sociedades desarrolladas actuales. Con el trabajo doméstico comparte invisibilidad social y una naturalizada adscripción al género femenino, pero se distingue y se desgaja de él por el acento en la dimensión relacional, de "persona a persona", del trabajo de cuidados. Un rasgo que permitirá ampliar su alcance para abarcar la provisión de servicios a las personas, dentro y fuera del hogar-familia. En ambos casos, las mujeres son las protagonistas del trabajo de cuidados. La división sexual del trabajo y la ideología que la sostiene, reproduce y otorga prestigios a tiempos y actividades, es vigente en las sociedades de capitalismo avanzado.

Desde perspectivas interesadas en las desigualdades de género, profundizar en el trabajo de cuidados en el hogar, ha permitido indagar en su carácter de hacer, de prever, de estar disponible para los demás y mostrar cómo este trabajo supone un notable esfuerzo de gestión cotidiana de actividades, ámbitos e instituciones sociales que traspasan las nociones de público-privado. Lejos de ser una actividad natural, los cuidados, su significado, las formas en que se dan, están atravesados por el género, la clase o la etnia. Los análisis en torno a la organización social del cuidado permiten conocer mejor cómo el trabajo de cuidados se conjuga en el triángulo estado-mercado-familia, así como las normas y valores que pautan y reproducen un desigual reparto de tareas y responsabilidades de cuidados.

Salir del círculo vicioso de los cuidados implica repensar qué es y qué significa cuidar y ser cuidado o cuidada. Significa plantear los cuidados como una responsabilidad colectiva y no como una cuestión individual y privada. Significa reclamar el derecho a unos servicios de cuidados accesibles y profesionalizados. Y significa, a su vez, reconocer material y simbólicamente el valor de los cuidados como pieza clave para el bienestar y la reproducción social.

\section{Bibliografía}

Abellán, A.; Esparza, C. y Pérez Díaz, V. (2011). Evolución y estructura de la población en situación de dependencia. Cuadernos de Relaciones Laborales. Vol. 29 Núm. 1, 43-67. 
Agulló, M ${ }^{\mathrm{a}}$.S. y Martín Palomo, $\mathrm{M}^{\mathrm{a}}$ T. (2011). Mayores cuidadores de mayores. Comunicación presentada al X congreso de la FES, celebrado en Pamplona, del $2-5$ de julio.

Annesley, C. (2007). Lisbon and social Europe: towards a European 'adult worker model' welfare system". Journal of European Social Policy [http://esp.sagepub.com/content/17/3/195].

Balbo, L. (1978). La doppia presenza. Inchiesta. Vol. VIII Núm. 32, 3-6.

Balbo, L. (Ed.) (1987). Time to care. Politique del tempo e diritti quotidiani. Milano: Franco Angeli.

Balbo, L.; May, M ${ }^{\mathrm{a}}$ P. y Micheli, G. A. (1990). Vincoli e strategie nella vita quotidiana. Milano: Franco Angeli.

Beck, U. (1992). Risck Society. Towards a New Modernity. London: Sage.

Béjar, H. (1988). El ámbito íntimo. Privacidad, individualismo y modernidad. Madrid: Alianza Universidad.

Benería, L. (2011). Crisis de los cuidados. Migración internacional y Políticas públicas. En C. Carrasco, C. Borderías y T. Torns (Eds.) El trabajo de cuidados. Historia, teoría y políticas (pp. 359-389). Madrid: La Catarata.

Benhabib, S. (1992). Una revisión del debate sobre las mujeres y la teoría moral. Isegoría. Núm. 16, 37-63.

Bettio, F.; Simonazzi, A. y Villa, P. (2006). Changes in care regimes and female immigration: the «care drain» in the Mediterranean. Journal of European social policy. Vol 16 Núm. 3, 271-285.

Bimbi, F. y Capecchi, V. (A cura di) (1986). Strutture e Strategie della vita quotidiana. Milano: Franco Angeli.

Borderías, C. (1996). Identidad femenina y recomposición del trabajo. En A. Rodríguez, B. Goñi y G. Maguregui (Eds.). El futuro del trabajo. Reorganizar y repartir desde la perspectiva de las mujeres ( $\mathrm{pp}$ 47-64). Bilbao: CEDEMBAKEAK.

Borràs, V.; Moreno, S. y Recio, C. (2009). La incorporación de los hombres en la esfera doméstica. Sociología del Trabajo. Núm. 67, 97-125. 
Borràs, V.; Torns, T. y Moreno, S. (2007). Las políticas de conciliación: políticas laborales versus políticas de tiempo. Papers. Núm. 83, 83-96.

Braidotti, R. (2000). Sujetos nómades. Barcelona: Paidós.

- (2004). Feminismo, diferencia sexual y subjetivismo nómade. Barcelona: Gedisa.

Brannen, J.; Moss, P, y Mooney, A. (2004). Working and Caring over the Twentieth Century. Hampshire: Palmgrave Macmillan.

Callejo, J; Prieto, C. y Ramos, R. (2008). Cambios generales en tiempo de trabajo profesional y de cuidados. En C. Prieto, R. Ramos y J. Callejo (Coords.). Nuevos tiempos de trabajo: Entre la flexibilidad competitiva de las empresas y las relaciones de género (pp 5-48). Madrid: CIS.

Cameron, C. y Moss, P. (2007). Care Work in Europe: Current understanding and future directions. Oxford: Routledge.

Carrasco, C. (2001). La sostenibilidad de la vida humana: ¿un asunto de mujeres? Mientras Tanto. Núm. 82, 43-70.

Carrasco, C.; Borderías, C. y Alemany, A. (1994). Las mujeres y el trabajo. Rupturas conceptuales. Barcelona-Madrid: Icaria-FUHEM.

Carrasco, C; Borderías, C. y Torns, T. (2011). El trabajo de cuidados. Madrid: La Catarata.

Carrasquer, P.; Torns, T.; Tejero, E. y Romero, A. (1998). El trabajo reproductivo. Papers. Núm 55, 95-114.

Castelló, L. (2011). Trabajo de cuidado, género y clase social. Remedios desiguales a un problema común. Sociología del Trabajo. Núm. 73, 24-41.

Chabaud-Rychter, D.; Fougeyrollas-Schwebel, D. y Sonthonnax, F. (1985). Espace et Temps du Travail domestique. Paris : Librerie des Méridiens.

Crompton, R. (2006). Employment and the family. Cambridge: Cambridge University Press.

Crompton, R. y Lyonette, C. (2010). Family, class and gender "strategies" in mothers' employment and childcare. En: J. Scott, R. Crompton y C. Lyonette (Eds.). Gender inequalities in the 21st century: New barriers and continuing constraints (pp 174-192). Cheltenham: Edward Elgar. 
Daly, M. y Lewis, J. (2000). The concept of social care and the analysis of contemporary welfare states. British Journal of Sociology. Vol. 51, Núm. 2, 281-298.

Daly, M. (2011). What Adult Worker Model? A Critical Look at Recent Social Policy Reform in Europe from a Gender and Family Perspective. Social Politics. Vol. 18. Núm.1, 1-23.

Del Re, A. (1995). Tiempo del trabajo asalariado y tiempo del trabajo de la reproducción. Política y Sociedad. Vol. 19, 75-81.

Dermott, R. (2005). Time and labour: Fathers perceptions of employment and childcare. The Sociological Review Vol 53, supl. 2, 89-103.

Duncan, S. (2011). The world we have made? Individualisation and personal life in the 1950s. The Sociological Review. Vol. 59 Núm 2, 242-265.

Durán, $\mathrm{M}^{\mathrm{a}} \mathrm{A}$. (2005). The future of work in Europe: Dependent care. En VVAA. Social Cohesion, Reconciliation Policies and Public Budgeting. A gender approach (pp. 41-73). Actas del International Experts Meeting October 24-26, 2005. México City.

Durán, Ma A. (Dir) (2011). El trabajo del cuidado en América Latina y España. Madrid: Fundación Carolina-CEALCI. Documentos de trabajo ${ }^{\circ} 54$.

England, P. (2005). Emerging theories of carework. Annual Review of Sociology. Núm. 31, 381-399. Doi:10.1146/annurev.soc.31.041304.122317.

Fine, M.; Glendinning, C. (2005). Dependence, independence or inter-dependence? Revisiting the concepts of 'care' and 'dependency'. Ageing \& Society. Núm. 25, 601-621. Doi:10.1017/S0144686X05003600.

Fraser, N. (1994). After the Family Wage: Gender Equity and Welfare State. Political Theory. Vol. 22, Núm. 4, 591-618.

Fraser, N. (2000). Rethinking recognition. New Left Review, Núm. 2, 107-120.

Gálvez, L.; Rodríguez, P. y Domíngez, M. (2011). Too much Family and Too Much Gender Inequality: Women's and Men's Total Work in Mediterranean Countries. En Addis, Degrave, de Villota y Eriksen (Eds.). Gender and well-being (pp 77104). Fanrham: Ashgate.

Giddens, A. (1992). The transformation of intimacy. Sexuality, Love \& Erotism in modern Societies. Cambridge - Oxford: Polity Press \& Blackwell. 
Gilligan, C. (1982). In a Different Voice. Cambridge: Harvard University Press.

Glenn, N. E. (2000). Creating a caring society. Contemporary Sociology. Vol. 29, Núm. 1, 84-94.

Graham, H. (1983). Caring: A Labour of Love. En J. Finch y D. Groves. A Labour of Love: Women, Work and Caring (pp. 13-30). London: Routledge \& Kegan Paul.

- (1991). The concept of Caring in Feminist Research: The Case of Domestic Service. Sociology, Núm. 25, 61-78.

Himmelweist, S. (2011). El descubrimiento del 'trabajo no remunerado'. En C. Carrasco, C. Borderías y T. Torns (Ed.). El trabajo de cuidados (pp 199-224). Madrid: La Catarata.

Hochschild, A. R. (1989). The second shift: Working Parents and the Revolution at Home. New York: Avon Books.

- (1995). The Culture of Politics: Traditional, Post-modern, Cold-modern, and Warmmodern Ideals of Care. Social Politics. Vol. 2. Núm. 3, 331-345.

Hobson, B. (2005). Madres ciudadanas, padres ciudadanos: las fronteras y los significados de las obligaciones y de los derechos se desplazan en la era del cambio en el estado del bienestar. En VVAA. SARE.2004. ¿Hacia qué modelo de ciudadanía? (pp 89-113). Vitoria-Gasteiz: EMAKUNDE-Instituto Vasco de la Mujer.

Izquierdo, $\mathrm{M}^{\mathrm{a}}$. J. (2004). Contenidos y significados de nuevas formas de cuidado. En VVAA. SARE.2003. Cuidar cuesta: costes y beneficios del cuidado. (pp. 119-154). Vitoria-Gasteiz: Emakunde-Fondo Social Europeo.

Lagarde, M. (2004). Las mujeres cuidadoras: entre la obligación y la satisfacción. En VVAA. SARE.2003. Cuidar cuesta: costes y beneficios del cuidado. (pp. 155-160). Vitoria-Gasteiz: Emakunde-Fondo Social Europeo.

Legarreta, M. (2008). El tiempo donado en el ámbito doméstico. Cuadernos de Relaciones Laborales, Vol. 26, Núm. 2, 45-69.

Letablier, M.T. (2007) El trabajo de "cuidados" y su conceptualización en Europa. En C. Prieto (ed.). Trabajo, género y tiempo social (64-84). Madrid: HacerUCM.

Lewis, J. (2009). Work-family balance, gender and policy. Cheltenham, UK: Edward Elgar. 
Lewis, J.D. y Weigert, A.J. (1992). Estructura y significado del tiempo social. En R. Ramos (Ed.). Tiempo y sociedad (pp 89-131). Madrid: CIS.

MacInnes, J. y Pérez Díaz, J. (2008). La tercera revolución de la modernidad: la revolución reproductiva. Revista Española de Investigaciones Sociológicas. Núm.122, 89-118.

- (2008). Domesticar el trabajo: una reflexión a partir de los cuidados. Cuadernos de Relaciones Laborales, Vol. 26, Núm. 2, 13-44.

Martín Palomo, $\mathrm{M}^{\mathrm{a}} \mathrm{T}$. (2010). Cuidados, género y generaciones: dilemas, retos y necesidades de las familias. Estudio etnográfico de tríadas generacionales a partir de la encuesta Redes Familiares en Andalucía. Sevilla: Instituto de Estadística de Andalucía.

McDowell, L.; Ray, K.; Perrons, D.; Fagan, C. y Ward, K. (2005). Women's paid work and moral economies of care. Social \& Cultural Geography, Vol. 6, Núm. 2, 219-235.

Moreno, S. (2007). Temps, treball i benestar: una aproximació des de la vida quotidiana. Tesis doctoral. Dep. Sociología- UAB.

Mosconi, N. y Paoletti, M. (2011). Le care: projet égalitaire ou cache misère? Travail, genre et societés, Núm. 26, pp. 173-177. Doi : 10.3917/tgs.026.0173.

Murillo, S. (1996). El mito de la vida privada. Madrid: Siglo XXI Ed.

- (2004). Cara y cruz del cuidado que donan las mujeres. En VVAA. Actas de las Jornadas "Cuidar cuesta: costes y beneficios del cuidado" (pp 161-176). Vitoria-Gasteiz: Emakunde-Fondo Social Europeo.

Nowotny, H. (1987). Il tempo: usi pubblici e usi privati. En L. Balbo. (Ed.). Time to care. Politique del tempo e diritti quotidiani (pp 15-23). Milano: Franco Angeli.

- (1992). Le temps à soi. Genèse et structuration d'un sentiment du temps. Paris: Editions de la Maison des sciences de l'homme.

Parella, S. (2003). Mujer, inmigrante y trabajadora: la triple discriminación. Barcelona: Anthropos.

Parker, R. (1981). Tending and social Policy. En E. M. Goldberg y S. Hatch (Eds.). A new look at the Social Services (pp 17-34). London: Policy Studies Institute. Documento de debate $n^{\circ} 4$. 
Paterman, C. (1995). El contrato sexual. Madrid: Anthropos.

Pérez Orozco, A. (2006). Amenaza tormenta: la crisis de los cuidados y la reorganización del sistema económico. Economía Feminista, Vol. 5, 7-37.

Pfau-Effinger, B. (1998). Gender Cultures and the Gender Arrangements- A Theoretical Framework for a Cross national gender research. Innovation. Vol.11, Núm. 2, 147-166.

Pocock, B. (2005). Work/Care Regimes: Institutions, Culture and Behaviour and the Australian Case. Gender, Work and Organization. Vol. 12 Núm. 1, 32-49.

Prieto, C. (2000). Trabajo y orden social: de la nada a la sociedad de empleo (y su crisis). Política y Sociedad. Vol. 34, 19-32.

- (2002). La degradación del empleo o la norma social del empleo flexibilizado. Sistema, Núm. 168-169, 89-106.

- (2004). Sentido y valor del tiempo de trabajo profesional, Género y representaciones de la 'vida personal'. Trabajo: Revista andaluza de Relaciones Laborales, Núm. 13, 37-58.

- (Ed.) (2007). Trabajo, género y tiempo social. Madrid: Editorial ComplutenseEditorial Hacer.

Puga González, Ma.D. y Abellán García, A. (2004). El proceso de discapacidad. Un análisis de la Encuesta sobre discapacidades, deficiencias y estado de salud. Alcobendas (Madrid): Fundación Pfizer.

Ramos R. (2011). Más allá de las cifras: la dimensión teórica y cualitativa del cuidado. En $\mathrm{M}^{\mathrm{a}}$ A. Durán (Dir.). El trabajo del cuidado en América Latina y España (pp 75-87). Madrid: Fundación Carolina-CEALCI. Documentos de trabajo $\mathrm{n}^{\mathrm{o}} 54$.

Recio, C. (2010). Familismo, Asistencialismo y Precariedad. La configuración del empleo en el sector de atención a las personas en España. Alternativas, Núm. 17, 19-43.

Rodríguez Cabrero, G. (2011a). Presentación. Cuadernos de Relaciones Laborales Vol. 29 Núm. 1, pp-11-12.

- (2011b). Políticas sociales de atención a la dependencia en los Regímenes de Bienestar de la Unión Europea. Cuadernos de Relaciones Laborales Vol. 29 Núm. 1, pp-13-42. 
Rogero García, J. (2010). Los tiempos del cuidado: el impacto de la dependencia de los mayores en la vida cotidiana de sus cuidadores. Madrid: Ministerio de Sanidad y Política Social- Imserso. Col. Estudios.

Saraceno, C. (1986). Uomini e donne nella vita quotidiana. Ovvero: per una analisi delle strutture di sesso della vita cuotidiana. En F. Bimbi y V. Capecchi (A cura di). Strutture e Strategie della vita quotidiana (pp 121-138). Milano: Franco Angeli.

- (2005). ¿Qué derechos y obligaciones, qué tipos de recursos? Visiones de la ciudadanía a través del prisma del género. En VVAA. SARE.2004. ¿Hacia qué modelo de ciudadanía? (pp 255-278). Vitoria-Gasteiz: EMAKUNDE-Instituto Vasco de la Mujer.

Sarasa, S. (2003). Una propuesta de servicios comunitarios de atención a personas mayores. Madrid: Fundación Alternativas. Documento de trabajo $\mathrm{n}^{\mathrm{o}} 15$.

- (2004). El descenso de la natalidad y los servicios de protección social a los ancianos. ICE, Núm. 815, 205-217.

Simonazzi, A. M. (2007). Care regimes and national employment model. Cambridge Journal of Economics. Núm. 33, 211-232.

Thomas, C. (1993). De-constructing concepts of care. Sociology, Vol. 27, Núm. 4, 649-669.

Tobío, C. (2012). Cuidado e identidad de género. De las madres que trabajan a los hombres que cuidan. Revista Internacional de Sociología [online], manuscrito aceptado. Doi: 10.3989/ris.2010.08.26.

Tobío, C.; Agulló, Ma S.; Gómez, M. V. y Martín Palomo, Ma T. (2010). El cuidado de las personas. Un reto para el siglo XXI. Barcelona: Fundació "La Caixa". Col. Estudios Sociales, $\mathrm{n}^{\mathrm{o}} 28$.

Torns, T. (2001). El tiempo de trabajo de las mujeres: entre la invisibilidad y la necesidad. En C. Carrasco (Ed.). Tiempos, trabajos y género (pp 133-147). Barcelona: Publicacions de la Universitat de Barcelona.

- (2004). Las Políticas de tiempo: un reto para las políticas del estado del bienestar. Trabajo: Revista andaluza de Relaciones Laborales. Núm. 13, 145-164.

- (2007). El tiempo de trabajo y las relaciones de género: las dificultades de un cambio ineludible. En C. Prieto (ed.). Trabajo, género y tiempo social (pp 269278). Madrid: Editorial Complutense- Editorial Hacer. 
- (2008). El trabajo y el cuidado: cuestiones teórico-metodológicas desde la perspectiva de género. EMPIRIA. Revista de Metodología de Ciencias Sociales. Núm. 15, 53-73.

Tronto, J. (2005). Cuando la ciudadanía se cuida: una paradoja neoliberal del bienestar y la desigualdad. En VVAA. SARE.2004. ¿Hacia qué modelo de ciudadanía? (pp 231-253). Vitoria-Gasteiz: EMAKUNDE-Instituto Vasco de la Mujer.

Ungerson, C. (1983). Why do Women Care? En J. Finch y D. Groves. A Labour of Love: Women, Work and Caring (pp 31-49). London: Routledge \& Kegan Paul.

-. (Eds.) (1990). Gender and Caring: Work and Welfare in Britain and Scandinavia. London: Harvester Whetssheaf.

- (2005). Care, work and feeling. Sociological Review. Vol. 53. Supl. 2, 188-203.

Vega, C. (2009). Culturas del cuidado en transición. Espacios, sujetos, imaginarios en una sociedad en migración. Barcelona: UOC.

VVAA. (2004). SARE.2003. Cuidar cuesta: costes y beneficios del cuidado. Vitoria-Gasteiz: EMAKUNDE-Fondo Social Europeo.

- (2005). SARE.2004. ¿Hacia qué modelo de ciudadanía? Vitoria-Gasteiz: EMAKUNDE-Instituto Vasco de la Mujer. 